\title{
Multirelaxation-time interaction-potential-based lattice Boltzmann model for two-phase flow
}

\author{
Zhao Yu (虞㻇) and Liang-Shih Fan* \\ Department of Chemical and Biomolecular Engineering, The Ohio State University, Columbus, Ohio 43210, USA
}

(Received 15 February 2010; revised manuscript received 26 July 2010; published 15 October 2010)

\begin{abstract}
The application of the lattice Boltzmann method in two-phase flows is often restricted by the numerical instability at low viscosities. In this work, a multirelaxation-time (MRT) lattice Boltzmann model (LBM) is developed using the interaction potential approach. With the MRT collision term and a general force term, the new MRT model is able to significantly enhance the numerical stability at low viscosities, without appreciable increase in computation time or memory use. Advanced force formulation using the multirange potential can also be readily incorporated into the current MRT scheme. Numerical tests are first performed in two dimensions under equilibrium conditions. The MRT model is able to reduce the lowest stable viscosity by an order of magnitude compared to the single relaxation time LBM. In addition, the spurious velocity at the gas-liquid interface can also be significantly decreased by tuning the adjustable relaxation parameters. Then two sets of three-dimensional simulations are conducted to investigate the buoyant rise of a gas bubble in a low-viscosity liquid. In particular, millimeter air bubble in water, which is difficult for traditional two-phase LBM due to both low viscosity and high-surface tension, is successfully simulated using the MRT technique developed in this study. The simulated bubble shape and velocity are compared with the experimental results and empirical correlations in the literature, and the satisfactory agreement proves the validity of the MRT-LBM for two-phase flows.
\end{abstract}

DOI: 10.1103/PhysRevE.82.046708

PACS number(s): 47.11.Qr, 47.55.dd

\section{INTRODUCTION}

In recent years, the lattice Boltzmann method (LBM) has attracted much attention as a powerful tool in direct numerical simulation of multiphase flows. Unlike other traditional computational fluid dynamics (CFD) techniques that solve the Navier-Stokes equation, the LBM employs the discrete Boltzmann equation which describes the fluid motion on the mesoscopic level. This kinetic nature provides the LBM with both clear physical concept and simple algorithm. In addition, for multiphase flows, the phase segregation in LBM can emerge naturally as the consequence of particle interactions, and therefore avoids the need to dynamically reconstruct or track the interface, which is required by many other multiphase simulation techniques such as volume of fluid, level set, and front tracking methods. Therefore, the LBM is becoming an increasingly popular method for multiphase flow simulations, and several types of two-phase LBM techniques have been developed. Among them, the most widely used ones are the interaction potential approach [1], the freeenergy approach [2], and the index-function approach [3]. Numerical techniques such as the finite difference scheme have also been applied in isothermal and thermal LBM models for gas-liquid systems $[4,5]$. In the meantime, a variety of multiphase flow problems have been investigated using LBM, such as bubble dynamics [6], droplets collisions [7], and two-phase flow in microfluidic devices [8].

However, there are also some limitations that are associated with LBM and restrict its applications. One well-known problem is that the LBM algorithm tends to become numerically unstable at low fluid viscosities. This problem becomes even worse in multiphase flow simulations, since lower vis-

\footnotetext{
*Corresponding author. fan.1@osu.edu
}

cosity often leads to larger spurious velocity at the interface, which destabilizes the interface and ultimately cause the computation to diverge. As a result of the instability problem, the parameter space in multiphase LBM simulation is often limited.

The numerical instability of LBM has received considerable attention in recent studies, and a number of techniques have been proposed to overcome this problem. Some researchers attribute the instability of LBM to the nonexistence of the $\mathrm{H}$ theorem, and accordingly they developed the entropic LBM by introducing the entropy function and a variable relaxation time [9]. The entropic LBM has shown better stability in several single phase flow simulations. However, its extension to multiphase flow problems has not been reported so far. The second type of techniques uses an implicit formulation of the collision term, which is treated using a central difference in both space and time [6]. Due to its implicitness, this scheme requires some iterative calculations, but the increase in computation cost is reported to be only moderate compared to the standard explicit algorithm. The implicit scheme has achieved enhanced stability at lowviscosity values, and is able to simulate bubbles with Reynolds number up to 400. The third class of techniques is based on the multiple-relaxation-time (MRT) scheme, which employs multiple relaxation parameters in the collision step, instead of the single-relaxation-time BGK collision in traditional LBM. During the collision step, the particle distribution functions are converted into their moments, which correspond to various hydrodynamic quantities and their fluxes that can relax on different time scales. There are a number of advantages of the MRT method, including enhanced numerical stability, the ability to model complex fluids such as viscoelastic flows, and ability to model thermohydrodynamics with adjustable Prandtl numbers [10]. In particular, a number of the two-phase models have been reformulated with the 
MRT algorithm. For instance, Tolke et al. [11] used an MRT version of the Gunstensen model [12] together with adaptive mesh to simulate the buoyant rise of a bubble in liquid. The incompressible two-phase flow model based on the index function [3] has been recast with MRT in both 2D [13] and 3D [14]. The MRT pressure-evolution model for high density ratio two-phase flows has also been developed [15]. It was able to increase the maximum accessible Reynolds number by $50 \%$ compared to the original single-relaxation version developed by [16]. The free-energy two-phase MRT model and the phase-field MRT model have been reported recently. These MRT models were shown to achieve much lower viscosities than their BGK versions [17]. The interaction potential model for nonideal gas [1] has also been recently extended with MRT algorithm [18], and was reported to achieve moderate improvement in stability. However, only simple flow conditions such as static droplets in equilibrium and capillary waves were simulated. It is noted that the MRT multiphase models typically inherit many numerical characteristics of the respective BGK versions from which they are derived. For example, the existing MRT models based on the index function have a better capability in preserving a sharp interface, while they are usually used for lower surface tension flows compared to the interaction potential model.

From the application point of view, in spite of the numerous studies on the development of LBM techniques, successful application of LBM in real-world gas-liquid two-phase flow problems is still quite limited. In fact, its instability at low-viscosity values is an important reason that restricts the application of LBM. In addition, high-surface tension in some systems also contributes to the numerical instability of multiphase LBM models. The consequence of the limitation in the parameter range can be illustrated in the example of a gas bubble rising in a viscous liquid, which is often characterized by the dimensionless groups Morton number (Mo) and Reynolds number (Re):

$$
\begin{gathered}
\text { Mo }=\frac{g \rho^{3} \nu^{4}}{\sigma^{3}}, \\
\operatorname{Re}=\frac{u_{b} d_{e}}{\nu},
\end{gathered}
$$

in which $g$ is the gravitational acceleration, $\rho$ and $\nu$ are the density and kinematic viscosity of the liquid, $\sigma$ is the surface tension, $u_{b}$ is the terminal rise velocity of the bubble, and $d_{e}$ is the equivalent bubble diameter. The most common system of an air bubble rising in water has a Morton number of $\mathrm{O}\left(10^{-11}\right)$, and millimeter-size air bubbles rising in water usually have a Reynolds number on the order of $10^{2} \sim 10^{3}$. Unfortunately, with its instability for low-viscosity fluids, the LBM is often limited to higher Mo $\left(\mathrm{Mo}>10^{-5}\right)$ and lower $\operatorname{Re}\left(\operatorname{Re}<10^{2}\right)$ conditions. For example, Frank et al. [19] used the free-energy LBM approach to simulate millimeter bubbles in $99.5 \%$ glycerol, which gave a Morton number of $\mathrm{O}\left(10^{1}\right)$ and $0.033<\operatorname{Re}<1.8$. A modified free-energy model with pressure Poisson equation [7] was used to simulated bubbles with $\mathrm{Mo}=10^{-5}$, although at a high density ratio of $10^{3}$. A phase field LBM for immiscible fluids was also ap- plied for bubbles with $10^{-5}<\mathrm{Mo}<10$, and $10^{-1}<\operatorname{Re}<10^{2}$ [20]. For the interaction potential LBM approach, similar applicable range is achieved, as shown by the coarse bifurcation studies $\left(\mathrm{Mo} \sim 10^{-4}\right)[21]$ and multiple bubble dynamics studies $\left(10^{-6}<\mathrm{Mo}<10^{2}, 10^{-1}<\operatorname{Re}<10^{2}\right)$ [22]. Recently the phase-field MRT approach was shown to achieve Mo $\sim 10^{-10}$ for two fluids with same densities, and Mo $\sim 10^{-8}$ at a density ratio of 3 . The highest reported $\mathrm{Re}$ in their work was about 300 for bubbles and 1000 for droplets [17]. To the best of the authors' knowledge, the closest LBM simulation for millimeter air bubbles in water to date was based on the implicit LBM formulation [6], which achieved a Morton number of $10^{-10}$, and Reynolds number up to 400 . However, even that does not match the conditions of the air-water system exactly. Therefore, an apparent gap exists between the capability of current two-phase LBM models and the actual flow conditions in many low-viscosity liquids, and there is clearly a demand to further improve the LBM model.

The motivation of the current work is to develop a new interaction potential based two-phase LBM model to address the numerical instability problem in high-surface tension, low-viscosity flows (low Mo, high Re). By using a slightly different way to incorporate the interaction force, the interaction potential model is integrated into the MRT algorithm in a straightforward manner. Formulation of the interaction force, such as the utilization of midrange potential, can be readily adapted in the developed model. The flexibility gained from the MRT algorithm and the midrange potential can be used to tune the stability property of the model. As the result, significant improvement of numerical stability and greatly reduced spurious velocity are found with the new model.

The rest of the paper is organized as follows. The MRTLBM will be presented in detail in Sec. II. The general framework of LBM will be briefly introduced first, followed by a discussion of the interaction force in the interaction potential model. Different techniques to incorporate the interaction force into the LBM are also described, including the shifted velocity approach in the original interaction potential model, and the approach with a separate force term. Then the solution of the newly developed MRT interaction potential model is described, with the emphasis on the transformation between the velocity space and the moment space during the collision step. Section III will be devoted to discuss the numerical results obtained from the new MRT interaction potential model. First the phase equilibrium in the MRT model will be analyzed in comparison to that in the BGK model. The improvement of the new MRT model will be evaluated regarding both the numerical stability and spurious velocity. Then the buoyant rise of gas bubbles in lowviscosity liquids will be simulated in three dimensions (3D) to test the model's performance in dynamic problems. Finally, summary of the model and the results will be provided in Sec. IV.

\section{MRT INTERACTION POTENTIAL MODEL}

\section{A. General framework of LBM}

The LBM is based on the Boltzmann equation, which can be written as 


$$
\frac{\partial f}{\partial t}+\mathbf{v} \frac{\partial f}{\partial \mathbf{x}}+\frac{\mathbf{F}}{\rho} \frac{\partial f}{\partial \mathbf{v}}=\Omega,
$$

where $f(\mathbf{x}, \mathbf{v}, t)$ is the particle distribution function, and $\mathbf{x}, \mathbf{v}$, $t, \rho, \mathbf{F}$ are the spatial coordinates, particle velocity, time, fluid density, and force, respectively. The right hand side of Eq. (3), $\Omega$, is the collision term that describes the change of particle distribution function due to particle collisions. In the LBM, the velocity space is discretized into a finite set of velocities $\left\{\mathbf{c}_{i}\right\}$ corresponding to a regular lattice structure in space, and accordingly the particle distribution is discretized into $\left\{f_{i}(\mathbf{x}, t)\right\}$. The collision is described as a relaxation process toward local equilibrium. The equilibrium distribution $f_{i}^{e q}(\rho, \mathbf{u})$ is a truncated Maxwell distribution that only depends on local fluid density $\rho$ and velocity $\mathbf{u}$, which can be calculated directly from the distribution functions $f_{i}$. The traditional LBM with the BGK collision term uses a single relaxation time scale $\tau$,

$$
\Omega=-\frac{f-f^{e q}}{\tau} .
$$

This relaxation parameter is in fact related to the kinematic viscosity of the fluid, as described by

$$
\nu=\left(\tau-\frac{1}{2}\right) c_{s}^{2} \Delta t
$$

However, in general the collision process involves multiple physical quantities that may relax on different time scales, and information for those time scales can be given using a full constant matrix $\boldsymbol{\Lambda}$ instead of a single time scale $\tau$,

$$
\Omega_{i}=-\sum_{j} \Lambda_{i j}\left(f_{j}-f_{j}^{e q}\right) .
$$

As the result, the LBE in the general form can be expressed as

$$
f_{i}\left(\mathbf{x}_{i}+\mathbf{c}_{i} \Delta t, t+\Delta t\right)-f_{i}\left(\mathbf{x}_{i}, t\right)=-\left.\sum_{j} \Lambda_{i j}\left(f_{j}-f_{j}^{e q}\right)\right|_{(x, t)}+\Delta t S_{i},
$$

The left hand side of Eq. (7), which is often referred to as the streaming or propagation step, corresponds to the unsteady and convective terms in the Boltzmann Eq. (3). The right hand side of Eq. (7) is referred to as the collision step. The last term $S_{i}$ in Eq. (7) represents the force term $\frac{\mathbf{F}}{\rho} \frac{\partial f}{\partial \mathbf{v}}$ in Eq. (3), and its implementation will be discussed in more detail in later sections. By convention, the lattice spacing and the time step are both normalized, so that $\Delta t=1$ and $\Delta x=c \Delta t$ $=1$. This convention is also adopted in this work.

Equation (7) provides the basic working equation for general LBM algorithms. It is noted that traditional LBM with BGK collision can be regarded as a special case in which the collision matrix is a diagonal matrix with identical diagonal elements of $1 / \tau$,

$$
\Lambda_{i j}=\frac{1}{\tau} \delta_{i j}
$$

\section{B. Interaction potential model}

The interaction potential model, also called the ShanChen model [1], is one of the most widely used multiphase LBM models. It employs a mean-field interaction force to mimic the molecular interactions that cause the segregation of the gas and liquid phases. An interaction potential $\psi$ is defined based on the local fluid density, and the interaction force is calculated from the interaction potential to induce proper phase separation. Two versions of interaction models have been developed: the single-component model for a nonideal fluid, and the multicomponent model for a mixture of two different fluids.

In the single-component model, the interaction force is given as the summation of the pairwise interactions between particles at a given lattice site and those at neighboring sites, and can be written as

$$
\mathbf{F}(\mathbf{x})=-G \psi(\mathbf{x}) \sum_{i=1}^{q} w_{i} \psi\left(\mathbf{x}+\mathbf{c}_{i}\right) \mathbf{c}_{i} .
$$

In the above equation, $G$ is a scalar constant that represents the strength of the interaction. The interaction potential $\psi$ is a local quantity that depends on the fluid density. In this study, the following form of the potential is employed [1],

$$
\psi(\rho)=1-\exp (-\rho) .
$$

Upon Taylor expansion of Eq. (9), the force can be written as

$$
\mathbf{F}=-\nabla\left(\frac{1}{2} c_{s}^{2} G \psi^{2}\right)-\frac{1}{2} c_{s}^{2} G \psi \nabla \nabla^{2} \psi+o\left(\Delta t^{3}\right),
$$

in which the first term on the right hand side corresponds to the nonideal part of the EOS, while the second term contributes to the surface tension. More detailed effects of the interaction force on the thermodynamic properties of the gasliquid interface are provided in a recent work by Shan [23] by considering the discrete lattice effect. Using the approach developed in that study, the properties of the gas-liquid interface can be found analytically, including the equilibrium densities, stress tensor, density profile across the interface, and the surface tension coefficient.

In the two-component model, two distributions $f_{i}^{\sigma}$ ( $\sigma$ $=1,2$ ) are used for the two components, and they each evolve according to the governing LB Eq. (7). The interaction force now includes both the interaction between particles of the same component and the particles of different components,

$$
\mathbf{F}_{\sigma}(\mathbf{x})=-\sum_{\bar{\sigma}=1}^{2} G_{\sigma \bar{\sigma}} \psi_{\sigma}(\mathbf{x}) \sum_{i=1}^{q} w_{i} \psi_{\bar{\sigma}}\left(\mathbf{x}+\mathbf{c}_{i}\right) \mathbf{c}_{i},
$$

The interaction strength parameters $\mathrm{G}_{11}$ and $\mathrm{G}_{22}$ describe the interaction within each individual component. When modeling a gas-liquid system, usually the first component is assumed to be a nonideal fluid with EOS determined by a potential as the one given in Eq. (10), while the second component is an ideal gas with $\mathrm{G}_{22}=0$ and $\psi_{2}\left(\rho_{2}\right)=\rho_{2} \cdot \mathrm{G}_{12}$ and $\mathrm{G}_{21}$ have the same value, and they describe the interaction between the two components and control the immiscibility of the mixture. 
In the original interaction potential model [1], only the first layer of neighbor sites are employed in the calculation of the interaction force. However, recent progress shows that several favorable features can be obtained by calculating the interaction force with the midrange potential, which involves an enlarged set of grid points in more layers of neighbor sites $[24,25]$. First, the extra degree of freedom leads to a surface tension that can be adjusted independently from the equation of state. Second, the magnitude of the spurious velocity can be reduced by either enlarging the interface thickness, or by employing a higher-order isotropic discretization. Since it is only involved in the formulation of the interaction force, the midrange potential can be readily integrated into the MRT model as well as the traditional interaction potential model.

\section{Incorporation of the force term}

In the original interaction potential model with BGK collision, the force term $\Delta t S_{i}$ in Eq. (5) is absorbed into a modified equilibrium distribution $\tilde{f}_{j}^{e q}\left(\rho, \mathbf{u}^{e q}\right)$, and the governing equation can be rewritten as

$$
f_{i}\left(\mathbf{x}_{i}+\mathbf{c}_{i} \Delta t, t+\Delta t\right)-f_{i}\left(\mathbf{x}_{i}, t\right)=-\frac{1}{\tau}\left(f_{i}-\tilde{f}_{i}^{e q}\right) .
$$

The equilibrium distribution is a function of a new equilibrium velocity, which is shifted by a quantity proportional to the force:

$$
\mathbf{u}^{\mathrm{eq}}=\overline{\mathbf{u}}+\frac{\tau \mathbf{F}}{\rho}
$$

$\overline{\mathbf{u}}$ is the velocity before the force is added. For example, in the single-component model,

$$
\rho \overline{\mathbf{u}}=\sum_{i} f_{i} \mathbf{c}_{i} .
$$

It can be shown that when the shifted velocity is used to calculate the equilibrium distributions, it introduces a momentum change proportional to $\mathbf{F}$ during the collision step.

Incorporating the force by shifting the equilibrium velocity, which is called the Shan-Chen forcing scheme, is a simple and unique feature of the original pseudopotential model. However, although the Shan-Chen forcing scheme is easy to implement with the BGK collision, its extension to the MRT framework is not straightforward. When multiple relaxation parameters are involved in the MRT algorithm, how to shift the velocity becomes a problem. Although a simple approach still using Eq. (14) has been reported [26], the theoretical basis for such shifting and the compatibility of such LB algorithm with the Navier-Stokes equation has not been discussed. Recently, a more rigorous approach using eigenvector decomposition has also been developed [18]. However, the algorithm is much more complicated than the original BGK model, and the resulted performance improvement is only marginal.

On the other hand, the force term in the continuous Boltzmann equation can be approximated using the gradient of the equilibrium distribution [27],

$$
-\frac{\mathbf{F}}{\rho} \frac{\partial f}{\partial \mathbf{v}} \approx-\frac{\mathbf{F}}{\rho} \frac{\partial f^{e q}}{\partial \mathbf{v}}=\frac{(\mathbf{v}-\mathbf{u}) \cdot \mathbf{F}}{\rho R T} f^{e q} .
$$

With a finite set of discrete velocities, the forcing term in the LBM becomes

$$
S_{i}=\frac{\left(\mathbf{c}_{i}-\mathbf{u}\right) \cdot \mathbf{F}}{\rho c_{s}^{2}} f_{i}^{e q} .
$$

Then, following the derivation given by Premnath and Abraham [14], by using a transformation $\bar{f}_{i}=f_{i}-\frac{1}{2} S_{i} \Delta t$, the general LB Eq. (7) is written as

$$
\begin{aligned}
\bar{f}_{i}\left(\mathbf{x}+\mathbf{c}_{i} \Delta t, t+\Delta t\right)-\bar{f}_{i}(\mathbf{x}, t)= & -\left.\sum_{j} \Lambda_{i j}\left(\bar{f}_{j}-f_{j}^{e q}\right)\right|_{(x, t)} \\
& +\left.\sum_{j}\left(I_{i j}-\frac{1}{2} \Lambda_{i j}\right) S_{j}\right|_{(x, t)} \Delta t,
\end{aligned}
$$

in which $I_{i j}$ is the components of the identity matrix. Since Eq. (18) applies to both single and multiple relaxation time algorithms, incorporation of the interaction force into the MRT approach becomes straightforward. The macroscopic fluid density is calculated as in traditional LBM,

$$
\rho=\sum_{i} f_{i} .
$$

And as the result of the transformation, the momentum of the fluid is calculated from

$$
\rho \mathbf{u}=\sum_{i} f_{i} \mathbf{c}_{i}+\frac{1}{2} \Delta t \mathbf{F} .
$$

It is also noted that, when using a single relaxation time, Eq. (18) reduces to the forcing scheme proposed by Guo et al. [28].

The way to incorporate the force term into the lattice Boltzmann equation has significant impact on its accuracy and numerical stability. For example, the original Shan-Chen model [1] in which the force is added by shifting the equilibrium velocity using Eq. (14) has been found to yield viscosity-dependent phase equilibrium [29], and its effect will be further demonstrated in Sec. III A. The interaction force in Eq. (11) is in fact similar to that used in the mean field approach for nonideal gas developed in [27], which expresses the force as $\mathbf{F}=-\nabla\left(p-\rho c_{s}^{2}\right)+k \rho \nabla \nabla^{2} \rho$. Direct discretization of the first gradient term, however, often leads to numerical instability near the interface, and this motivates the development of the index-function method [3]. In the index-function approach, by transformation of the distribution functions, the first gradient term is multiplied by a small quantity $[\Gamma(0)-\Gamma(\mathbf{u})]$, which effectively reduces the magnitude of the forcing term and therefore greatly improves the numerical stability. On the other hand, the special form of discretization used in Eqs. (9) and (12) in Shan-Chen's approach contributes to the numerical stability of the interaction potential method, and the effects of such discretization and its extension have been analyzed in the literature [24,25]. Comparisons between the index-function MRT model and 
the current interaction potential MRT mode in terms of numerical stability at different surface tension and viscosity values are provided in Secs. III C and III E.

\section{Solution of MRT-LBE}

After incorporating the force term, Eq. (18) becomes the governing equation that dictates the evolution of the distribution functions in MRT-LBM. Since the collision matrix $\boldsymbol{\Lambda}$ is in general a full matrix, directly solving Eq. (18) involves complex matrix manipulations. However, in practice, the solution of Eq. (18) takes advantage of a special linear transformation to diagonalize the collision matrix. The transformation matrix $\mathbf{T}$ transforms the distribution functions $\bar{f}_{i}$, which lie in the velocity space, into their moments $\hat{f}$, which correspond to macroscopic physical quantities such as density, momentum, energy, and their fluxes that lie in the moment space.

$$
\hat{f}=\mathbf{T} \bar{f} .
$$

The specific form of the transformation matrix depends on the lattice structure. The procedure presented in this section corresponds to the D2Q9 lattice in 2D. The 3D cases using D3Q19 lattice has been given in the reference [14]. With D2Q9 lattice, the transformed vector $\hat{f}$ in the moment space is given explicitly by

$$
\hat{f}=\left[\rho, e, e^{2}, j_{x}, q_{x}, j_{y}, q_{y}, p_{x x}, p_{x y}\right]^{T},
$$

where $e$ is the energy, $e^{2}$ is the energy squared; $j_{x}$ and $j_{y}$ are the momentum in $\mathrm{x}$ and $\mathrm{y}$ direction; $q_{x}$ and $q_{y}$ are the energy fluxes; $p_{x x}$ and $p_{x y}$ are the diagonal and off-diagonal components of the stress tensor. The transformation matrix is expressed explicitly as [10]

$$
\mathbf{T}=\left[\begin{array}{ccccccccc}
1 & 1 & 1 & 1 & 1 & 1 & 1 & 1 & 1 \\
-4 & -1 & -1 & -1 & -1 & 2 & 2 & 2 & 2 \\
4 & -2 & -2 & -2 & -2 & 1 & 1 & 1 & 1 \\
0 & 1 & 0 & -1 & 0 & 1 & -1 & -1 & 1 \\
0 & -2 & 0 & 2 & 0 & 1 & -1 & -1 & 1 \\
0 & 0 & 1 & 0 & -1 & 1 & 1 & -1 & -1 \\
0 & 0 & -2 & 0 & 2 & 1 & 1 & -1 & -1 \\
0 & 1 & -1 & 1 & -1 & 0 & 0 & 0 & 0 \\
0 & 0 & 0 & 0 & 0 & 1 & -1 & 1 & -1
\end{array}\right]
$$

The equilibrium moments are obtained by applying the transformation to the equilibrium distributions originally in the velocity space, and can be computed directly from the hydrodynamic quantities such as density and momentum.

$$
\hat{f}^{e q}=\mathbf{T} \bar{f}^{e q}=\left[\begin{array}{c}
\rho \\
-2 \rho+\left(j_{x}^{2}+j_{y}^{2}\right) / 9 \rho \\
\rho-\left(j_{x}^{2}+j_{y}^{2}\right) / 9 \rho \\
j_{x} \\
-j_{x} \\
j_{y} \\
-j_{y} \\
\left(j_{x}^{2}-j_{y}^{2}\right) / \rho \\
j_{x} j_{y} / \rho
\end{array}\right]
$$

In the same way, the forcing term is also transformed into the moment space.

$$
\hat{S}=\mathbf{T} S=\left[\begin{array}{c}
0 \\
6\left(u_{x} F_{x}+u_{y} F_{y}\right) \\
-6\left(u_{x} F_{x}+u_{y} F_{y}\right) \\
F_{x} \\
-F_{x} \\
F_{y} \\
-F_{y} \\
2\left(u_{x} F_{x}-u_{y} F_{y}\right) \\
u_{x} F_{y}+u_{y} F_{x}
\end{array}\right] \text {. }
$$

By multiplying the transformation matrix $\mathbf{T}$, the right hand side (RHS) of Eq. (18) can be entirely transformed into the moment space,

$$
\begin{aligned}
\mathbf{T}(\mathrm{RHS})= & -\left.\sum_{\beta} \hat{\Lambda}_{\alpha \beta}\left(\hat{f}_{\beta}-\hat{f}_{\beta}^{(e q)}\right)\right|_{(x, t)} \\
& +\left.\sum_{\beta}\left(I_{\alpha \beta}-\frac{1}{2} \hat{\Lambda}_{\alpha \beta}\right) \hat{S}_{\beta}\right|_{(x, t)} \Delta t .
\end{aligned}
$$

The transformed collision matrix $\hat{\Lambda}=\mathbf{T} \mathbf{\Lambda} \mathbf{T}^{-1}$ is now diagonal in moment space,

$$
\hat{\boldsymbol{\Lambda}}=\operatorname{diag}\left[s_{1}, s_{2}, s_{3}, s_{4}, s_{5}, s_{6}, s_{7}, s_{8}, s_{9}\right]
$$

The diagonal elements $s_{1}$ through $s_{9}$ are the new relaxation parameters associated with each components of $\hat{f}$. Since the new collision matrix is diagonal, the relaxations of different physical quantities to $\hat{f}^{e q}$ are now decoupled. Different time scales determined by $s_{1}$ through $s_{9}$ can be adjusted independently, although a few constraints still apply. To be consistent with the macroscopic hydrodynamic equation, it is required that $s_{1}=s_{4}=s_{6}=1$. Meanwhile, $s_{8}$ and $s_{9}$ are related to the kinematic viscosity $\nu$,

$$
s_{8}=s_{9}=1 / \tau \text {, and } \nu=\left(\tau-\frac{1}{2}\right) c_{s}^{2} \Delta t \text {. }
$$

And $s_{2}$ is related to the bulk viscosity of the fluid by 


$$
\zeta=\left(\frac{1}{s_{2}}-\frac{1}{2}\right) c_{s}^{2} \Delta t .
$$

Further, symmetry requires that $s_{5}=s_{7}$. As the result, three of the relaxation parameters $s_{2}, s_{3}, s_{5}\left(=s_{7}\right)$ remain to be independently adjustable, and they can be used to tune the stability of the MRT model.

In practice, the MRT-LBM algorithm is often realized in a way such that the collision step takes place in the moment space, while the propagation step still operates in the velocity space. In the actual computation procedure, transformed quantities $\hat{f}, \hat{f}^{e q}$, and $\hat{S}$ are first calculated using Eqs. (22), (24), and (25), followed by the collision step as described by Eq. (26). Then the RHS is transformed back to the velocity space to perform the propagation step,

$$
\bar{f}_{i}\left(\mathbf{x}+\mathbf{c}_{i} \Delta t, t+\Delta t\right)-\bar{f}_{i}(\mathbf{x}, t)=\mathbf{T}^{-1}[\mathbf{T}(\mathrm{RHS})] .
$$

The hydrodynamic quantities such as density, momentum, potential, and force are calculated in the velocity space in the usual way as discussed in previous sections.

In summary, the current MRT interaction potential method models the two-phase flow based on the interaction force calculated from the interaction potential, as in the classic Shan-Chen interaction potential model. However, the interaction force is directly incorporated into the LBM without shifting the equilibrium velocity. In the collision step, the particle distributions are transformed into their moments which have distinct physical meanings. Their relaxation time scales are uncoupled and can be adjusted independently with the parameters $s_{1} \sim s_{9}$. The separation of different relaxation scales brings improved stability to the MRT interaction potential model. In addition, since the transformation between velocity space and moment space is a local operation, the transformed moments $\hat{f}$ do not need to be stored. Therefore, the MRT algorithm only requires a small amount of additional computation time compared to conventional LBM, without significant increase in memory usage.

\section{NUMERICAL RESULTS}

\section{A. Phase equilibrium properties}

A unique feature of the interaction potential model is that the phase segregation is induced by particle interaction forces. Many thermodynamic properties such as the equilibrium density, equilibrium pressure, and surface tension are directly related to the way the interaction force is evaluated and incorporated into the lattice Boltzmann equation. In order to examine these equilibrium properties in the current MRT model, simulations are carried out in a $2 \mathrm{D}$ periodic domain, with 20 and 200 grids in the horizontal and vertical directions, respectively. The adjustable relaxation parameters are chosen to such that $s_{2}=0.3, s_{3}=1.5, s_{5}=s_{7}=1.2$. The computation is initialized with a slab of gas phase in the middle of the domain and liquid phase in other regions, and runs until the macroscopic velocity in the whole field becomes negligibly small, which characterizes the arrival of equilibrium. The equilibrium density and pressure in each phase are then extracted for analysis.
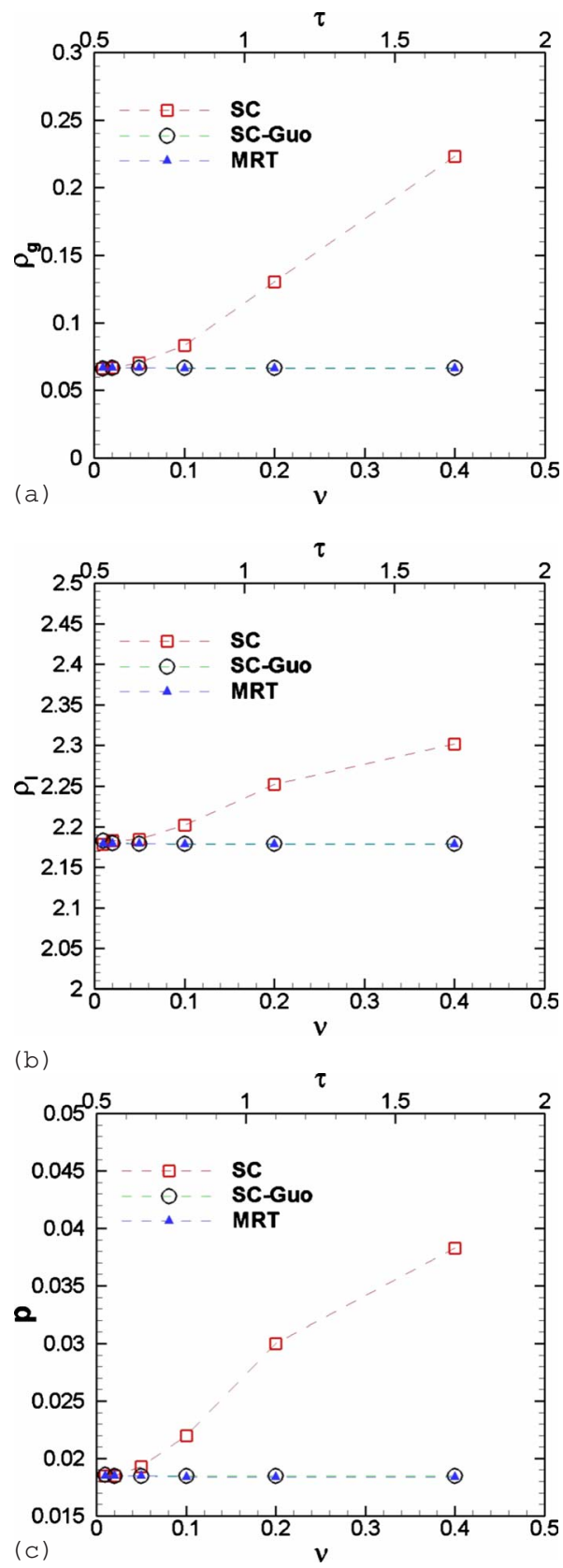

FIG. 1. (Color online) Effect of the relaxation time $\tau$ (and equivalently viscosity $\nu$ ) on the equilibrium properties in the original Shan-Chen interaction potential model (SC), the Shan-Chen model with Guo's forcing scheme (SC-Guo), and the current MRT scheme for a single-component fluid. Simulations are carried out at $G=-5.4$. (a) Effect of $\tau$ on gas phase density. (b) Effect of $\tau$ on liquid phase density. (c) Effect of $\tau$ on pressure.

Figure 1 shows the simulation results for the equilibrium density and pressure of a single-component nonideal fluid under the condition $G=-5.4$. The equilibrium properties at varying kinematic viscosity are presented for the MRT model as well as the original Shan-Chen (SC) model and the SCGuo model. The interaction force is calculated in the same way in all three models, by summation of interactions with only the first layer of eight neighbors on the D2Q9 lattice. Both the SC and SC-Guo models use the single relaxation 


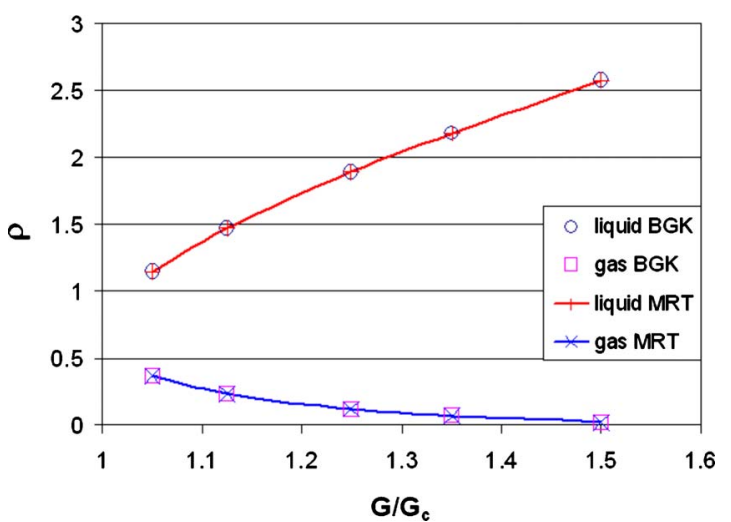

FIG. 2. (Color online) Phase diagram of a single-component fluid obtained from the SC-Guo (BGK) scheme and current MRT scheme. The densities of the liquid phase and the gas phase are plotted as functions of the normalized interaction strength $G / G_{c}$, where $G_{c}$ is the critical interaction strength.

(BGK) collision. Their difference is that the SC model incorporates the interaction force by shifting the velocity, while the SC-Guo uses the Guo's forcing scheme [27]. As shown in Fig. 1, the equilibrium properties predicted by both the SCGuo model and the MRT model remain nearly constant regardless of the change in viscosity, or equivalently, the relaxation parameter $\tau$. However, the results from the original Shan-Chen model show clear variation as viscosity changes. The variable showing most significant change is the gas phase density, which reduces about $60 \%$ as viscosity changes from 0.4 to 0.01 . It is noted that the results in Shan-Chen model tend to converge to the value predicted by the other two models only when the viscosity becomes very small. Such effect of viscosity on the equilibrium has been reported previously for the SC and SC-Guo models [27]. The coupling between equilibrium and viscosity is an unfavorable feature of the original interaction potential model, and is speculated to be related to its incorporation of the force by shifted velocity, which introduces a nonlinear error term that scales with $F^{2}$. The fact that the equilibrium properties are independent of the viscosity is clearly an attractive characteristic of the SC-Guo and MRT model, since it makes it convenient to specify fluid properties of the two phases.

The phase diagram of the single-component fluid can be obtained by carrying out the simulation for different values of $G$ used in the calculation of the interaction force in Eq. (9). The critical value of $G$ corresponding to the potential given in Eq. (10) is -4 , which can be calculated from the equitation of state. The phase diagrams simulated using the SC-Guo model and the MRT model are presented in Fig. 2. The two models predict almost identical equilibrium densities at various values of $G$, indicating that the MRT model does not alter the equilibrium properties of the original interaction potential model. The density ratio between the liquid and the gas phase ranges from 3 to 170 when $G$ changes from -4.2 to -6.0 . Although the two models predict the same equilibrium properties, the MRT model usually reaches equilibrium much faster than the SC-Guo model, especially at low viscosities. This faster convergence is probably related to the larger value of the bulk viscosity, which can be speci-

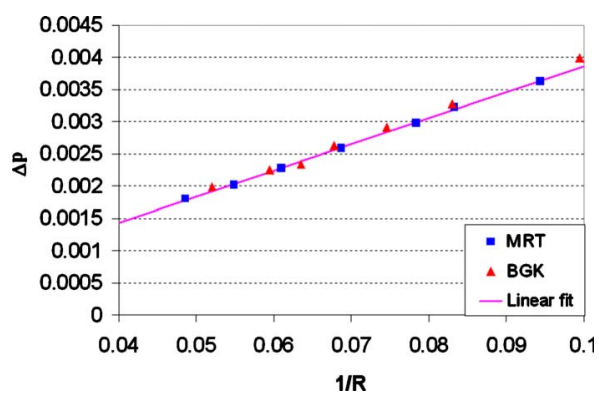

FIG. 3. (Color online) Laplace law tests for the SC-Guo model with BGK collision and the MRT model. The pressure difference across the interface is plotted against the curvature. Both models use the single-component model with the same interaction strength $G=-5.0$. The linear fit is made using the results obtained from the MRT model, and gives the surface tension coefficient of 0.041 .

fied independently from the shear viscosity in the MRT model.

\section{B. Surface tension}

Surface tension in the interaction potential model comes from the fourth order term in the Taylor expansion of the interaction force, and its theoretical value can be calculated from the stress profile across the interface [23]. In practice, the value of the surface tension is computed from the pressure difference across a circular interface according to the Laplace law:

$$
\Delta p=\sigma\left(\frac{1}{R_{1}}+\frac{1}{R_{2}}\right)
$$

In the above equation, $\Delta p, \sigma, R_{1}$, and $R_{2}$ are the pressure difference, surface tension coefficient, and the principal radii of curvature of the interface. Simulations for a singlecomponent fluid using both the SC-Guo model and the MRT model are performed for a number of circular bubbles with varying radii at equilibrium. In both models, the interaction force is computed form nearest neighbors, and the interaction strength has the value $G=-5.0$. The simulation results plotted in Fig. 3 show a linear relationship between the pressure drop and the curvature for both models, and the data obtained from the two models fall on a single line, indicating that the MRT approach does not change the surface tension value in the original pseudopotential model under the same conditions.

\section{Spurious velocity}

The spurious velocity, also referred to as the parasite current, is the small circulating velocity that exists near the interface in the numerical results. This spurious velocity is unphysical, and its presence not only degrades the accuracy of the simulation, but also causes instability problems. This numerical artifact is a common problem for many two-phase flow simulation techniques, including VOF, Level-set, and LBM [30]. The magnitude of the spurious velocity is found to increase as the surface tension value increases, or the interface width decreases. In the literature, the interaction po- 


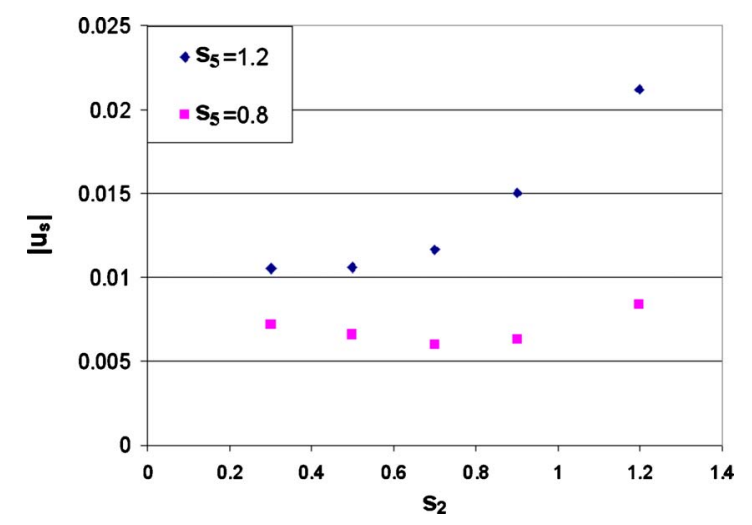

FIG. 4. (Color online) Effects of relaxation parameters $s_{2}$ and $s_{5}$ on the magnitude of the spurious velocity in the single-component MRT model. 2D simulations are performed with $G=-5.0, s_{3}=1.5$, and $\nu=0.02$ (i.e., $s_{7}=1.7857$ ).

tential model is reported to have a larger spurious velocity compared to the free-energy LBM, which is probably related to the fact that it often uses a higher surface tension value. In practice, the spurious velocity results in a serious limitation of the LBM in two-phase flow simulations, especially at high density ratios or low viscosities [31]. The original of the spurious velocity has been identified to be the insufficient isotropy in evaluating the gradient terms for force calculation [23].

Since the MRT scheme involves additional freely adjustable relaxation parameters, it may benefit from the extra flexibility to reduce the spurious velocity. The effect of the adjustable relaxation parameters in the current MRT model for a single-component fluid is demonstrated in Fig. 4. The numerical tests are carried out for 2D bubbles with $G=-5.0$ and $\nu=0.02$. Under such condition, the BGK algorithm is unstable due to severe spurious velocity. In the MRT simulation, two sets of numerical tests are conducted with $s_{5}$ $=1.2$ and $s_{5}=0.8$, respectively. In each of the tests, the value of $s_{2}$ is varied from 0.3 to 1.2 . While the magnitude of the spurious velocity increase monotonically with $\mathrm{s}_{2}$ when $s_{5}$ $=1.2$, it has a minimum value of 0.0062 at $s_{2}=0.7$ when $s_{5}$ $=0.8$. These tests demonstrate that adjusting the free relaxation parameters can effectively decrease the spurious velocity and make the simulation numerically stable. However, the effect of each parameter on the spurious velocity is found to be case dependent. In addition, the free parameters in 2D (D2Q9 lattice) and 3D (D3Q19 lattice) simulations also show different effects. Therefore, no universal rule to reduce the spurious velocity has been established at this moment. It should also be noted that physical properties of the fluid is not affected by adjusting these free parameters. For example, all the simulations in Fig. 4 give an almost identical surface tension value of 0.0378 , with the largest deviation to be within $2 \%$. This consistency in physical properties makes it convenient to use these adjustable relaxation parameters for tuning the stability of the computation.

Figure 5 presents the magnitude of the spurious velocity as a function of viscosity in three different versions of the single-component model. The spurious velocity shown is the maximum value measured from the numerical results for a

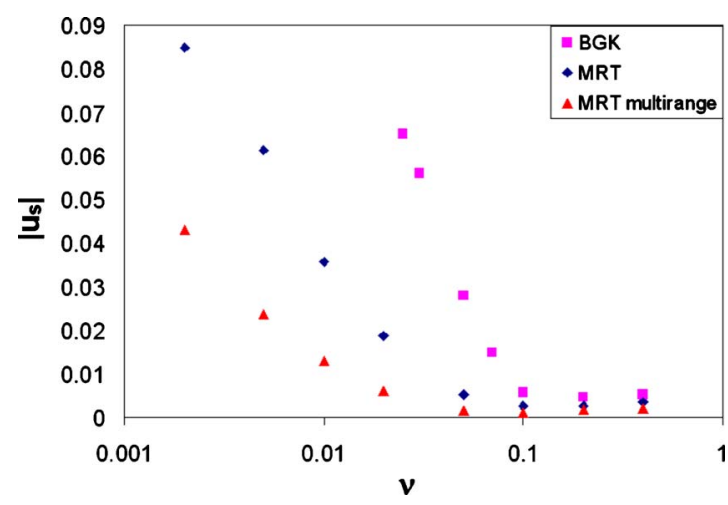

FIG. 5. (Color online) Magnitude of the spurious velocity as a function of viscosity in the single-component model. The SC-Guo model with BGK collision is unstable with viscosity less than 0.025 .

2D circular bubble at equilibrium. The spurious velocity increases as the viscosity decreases in all models. Especially, a sharp increase is found in the SC-Guo model when viscosity approaches 0.025 . The spurious velocity ultimately becomes so large that no stable results could be obtained for viscosity lower than 0.025 in the SC-Guo model. The MRT model with the same force evaluation gives consistently smaller spurious velocity compared to the BGK model. As a result, fluid with viscosity as low as 0.002 can be easily simulated using the MRT model.

It has been shown that the multirange potential can be used to reduce the spurious velocity in the interaction potential model. By utilizing more neighboring lattice sites in the evaluation of the potential gradient, two approaches have been proposed to mitigate the spurious velocity, including using high-order isotropic discretization [24] [25], and increasing the width of the interface [24]. It should be noted that adjustments in the multirange potential may have the effect of changing the surface tension [24]. This effect can be understood from Eq. (11), in which the coefficient in front of the term $\psi \nabla \nabla^{2} \psi$ is proportional to the square of the surface tension. A larger value of this coefficient corresponds to both higher surface tension, and wider interface. These two factors actually have opposite effects on the amplitude of the spurious velocity, but numerical results show that their combined effect is to decrease the spurious velocity.

The spurious velocity in the MRT scheme with midrange potential is also plotted in Fig. 5. The midrange potential model uses two layers of lattice neighbors ( 24 points) in the force calculation, and results in an interaction force that is isotropic up to the eighth order. In comparison, the original force evaluation using only the first layer of eight lattice points can only reach fourth order isotropic discretization [25]. The spurious velocity in the MRT-midrange model is consistently smaller than the MRT model under all viscosities, and its magnitude can often be reduced to below half of that in the MRT model with regular force evaluation method, as shown in Fig. 5.

The spurious velocity near a $2 \mathrm{D}$ circular interface is shown in Fig. 6. The three simulation are carried out under identical condition where $G=-5.0$ and $\nu=0.05$. The dilute phase inside the circle has a density of 0.11 , and the dense 



FIG. 6. (Color online) Spurious velocity at the gas-liquid interface in two dimensions. The same fluid properties are used in three simulations: $G=-5.0, \nu=0.05$. The gas and liquid densities are 0.11 and 1.85 , respectively. The interface shown in the figures are plotted with density contours of 0.5 and 1.0. The spurious velocity is plotted under the same magnification in the three figures. (a) BGK model. $\left|u_{s}\right|_{\max }=0.028$; (b) MRT model. $\left|u_{s}\right|_{\max }=0.0053$; (c) MRT model with eighth order isotropic force. $\left|u_{s}\right|_{\max }=0.0016$.

phase outside the circle has a density of 1.85 . The maximum amplitude of the spurious velocities in BGK model, MRT model, and MRT-midrange model are 0.028, 0.0053, and 0.0016, respectively. The vector plots Figs. 6(a)-6(c) clear demonstrates that the combination of MRT and midrange potential model can effectively reduce the spurious velocity. As in the original interaction potential model, an increase in the value of $G$ will lead to an increase in surface tension in the present MRT model, and hence an increase in the mag-

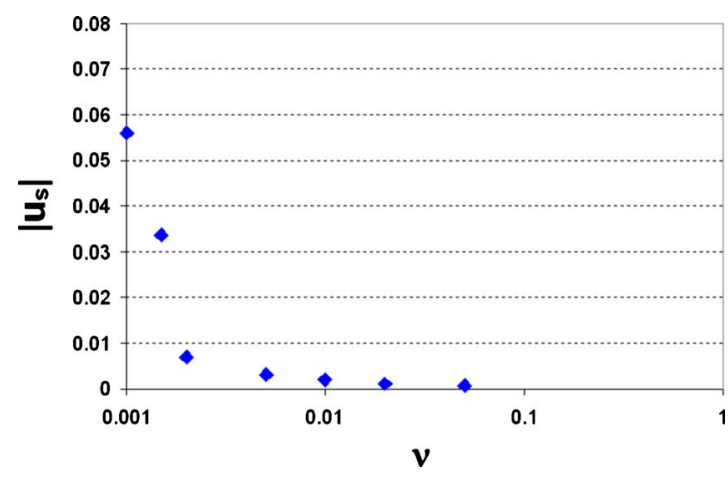

FIG. 7. (Color online) Magnitude of the spurious velocity as a function of viscosity in the two-component MRT model.

nitude of spurious velocity. However, the spurious velocity in the MRT model is observed to be consistently smaller than that in the BGK model when the same value of $G$ is used for both models.

For the two-component interaction potential MRT-LBM model, the magnitude of the spurious velocity can also be significantly reduced to enhance the numerical stability. Figure 7 shows the change of the spurious velocity magnitude at different kinematic viscosities in 3D simulations. The interaction strengths used are $G_{11}=5.0, G_{12}=G_{22}=0$. The adjustable relaxation numbers are $s_{2}=0.6, s_{3}=1.4, s_{5}=1.99, s_{11}$ $=0.1$, and $s_{17}=0.3$. Multirange potential is employed to include 84 neighbor lattice sites in the evaluation of the interaction force. The resulted distributions of the two components in the two phases are: $\rho_{1 g}=0.053, \rho_{2 g}=0.115, \rho_{1 l}$ $=1.945$, and $\rho_{2 l}=0.012$. As the viscosity value decreases from 0.05 to 0.001 , the magnitude of the spurious velocity monotonically increases from 0.0008 to 0.056 , as shown in Fig. 9. While the current MRT model is still stable under a viscosity value as small as 0.001 , in comparison, the original two-component interaction potential model with BGK collision typically diverges when viscosity falls below 0.02 .

In order to compare the current interaction potential MRT approach with existing two-phase MRT lattice Boltzmann methods, the equilibrium bubble simulations are also performed with the index-function based MRT technique, for which the implementation and the parameters employed have been given in detail in [13]. Compared to the interaction potential method, the index-function method is often found to yield a lower surface tension and a smaller density ratio. For $\nu=0.01, k=0.05$, and $\rho_{g}=0.1$, the highest stable density ratio obtained in the index-function MRT approach is found to be 10.2 . When the density ratio changes from 3 to 10 , the value of the surface tension varies accordingly from 0.00065 to 0.0145 , and thus the value of $\sigma / \rho_{l}$ changes from 0.0022 to 0.0145 . Although the surface tension can be adjusted by changing $k$, the stable range of $k$ is also limited, and the upper limit of $k$ decreases with higher density ratio. For example, at the density ratio of 4 , the largest value of $k$ is found to be 0.15 , which gives a surface tension of 0.0039 . In contrast, in the interaction potential MRT approach developed in this work, the value of $\sigma / \rho_{l}$ is typically in the range between 0.01 and 0.05 for the single-component model, and between 0.01 and 0.15 for the two-component model. Besides, the 
interaction potential approach also has a higher stable density ratio, which is about 40 for the single-component model and about 15 for the two-component model using the potential form given originally in [1]. Since the potential approach relies solely on the interaction force to realize phase separation, strong interaction force, and consequently higher surface tension, is often required in order to maintain a sharp interface. In fact, it often has difficulty to obtain small surface tension values and is less convenient for flows with high Weber number or Eotovos number. The magnitude of the spurious velocity in the two techniques is similar, and is both between $\mathrm{O}\left(10^{-3}\right)$ and $\mathrm{O}\left(10^{-2}\right)$ for stable simulations. The index-function MRT is found to be more stable than the interaction potential MRT model at extremely small viscosities. It is able to reach viscosity as low as 0.0001 at the density ratio of 4 , while the interaction potential MRT model generally becomes unstable for viscosities lower than 0.001 . In addition, the index-function model often results in a sharper interface compared to the potential model. The above numerical characteristics on the two models have some implications in their applications for multiphase flow problems, which will be further discussed in Sec. III E.

\section{Dynamic property}

In order to test the capability of the newly developed MRT approach for dynamic problems, the simulation of a capillary wave is conducted and compared to the analytical solutions obtained from the potential theory. The simulations are performed in a $2 \mathrm{D}$ periodic domain, and the initial interface profile is perturbed using a sinusoidal wave with small amplitude. The single-component MRT model was employed with $G=-5.5$. The densities of the two phases are 2.27 and 0.07 , respectively. The value of the surface tension measured from a static drop simulation is 0.086 . Oscillations of the interface at different times are plotted in Fig. 8(a) for two different viscosities. A higher viscosity leads to a faster decay of the wave amplitude, while it has no effect on the frequency, which is in accordance with the potential theory. Figure 8(b) shows the dispersion relation between the wave number $k=2 \pi / L$ and angular frequency $\omega$. The simulation results are compared to the analytical expression given by [18]

$$
\omega^{2}=\frac{\sigma}{\rho_{g}+\rho_{l}} k^{3} .
$$

The good agreement found in the figure indicates that the surface tension value obtained from the static drop simulation is the same as that from the dynamic capillary wave simulation. This fact further validates the accuracy of the present MRT model for both types of simulations.

\section{E. Buoyant rise of bubbles in liquid}

The buoyant rise of a millimeter-size gas bubble in a lowviscosity liquid is often characterized by small Morton number and high Reynolds number. Due to the improved numerical stability at small viscosities, the newly developed MRTLBM is able to successfully simulate such bubbles, which
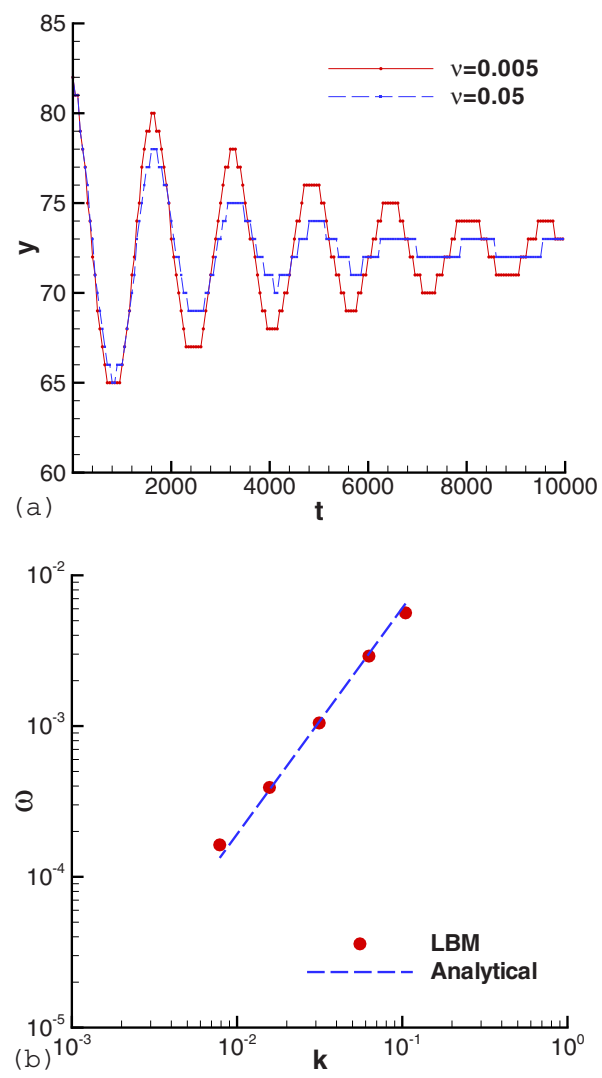

FIG. 8. (Color online) Capillary wave at the interface between two fluids. (a) Oscillation of the interface at different viscosities. The height of the capillary wave is plotted against time $t$. (b) Comparison between the simulated frequency $\omega$ and the analytical solution from potential theory at different wave number $k$.

are usually difficult for traditional two-phase LBM. The 3D bubble simulations are carried out using the two-component MRT model on D3Q19 lattice. The equivalent diameter of the gas bubble is about 30 lattice units. The simulation domain is $150 \times 150 \times 300$ lattice units in width, length, and height. Such domain size is believed to result in negligible boundary effect on the bubble dynamics. The simulation first runs without gravitational force for a few thousand steps to let the system equilibrate. Once the bubble volume reaches a constant value, the gravity is turned on and simulation continues to run until the bubble reaches a steady velocity. The simulation results are compared with experimental results or empirical correlations in the literature using the dimensionless groups Mo, Eo, and Re to validate current MRT-LBM model.

The first set of simulations are conducted at $\mathrm{Mo}=6.2$ $\times 10^{-7}$, corresponding to recent experiments of millimeter gas bubbles in silicone oil DMST-05 [32]. The equivalent bubble diameters are in the range between 1.5 and $3.5 \mathrm{~mm}$, which give the Eotvos numbers $\left(\mathrm{Eo}=\frac{\rho d^{2} g}{\sigma}\right)$ between 1.0 and 5.6. Figure 9(a) shows the Reynolds number of the bubbles at their terminal velocities. The simulation results are in good agreement with both the values measured in the experiment and the empirical correlation given by Fan and Tsuchiya [33], 

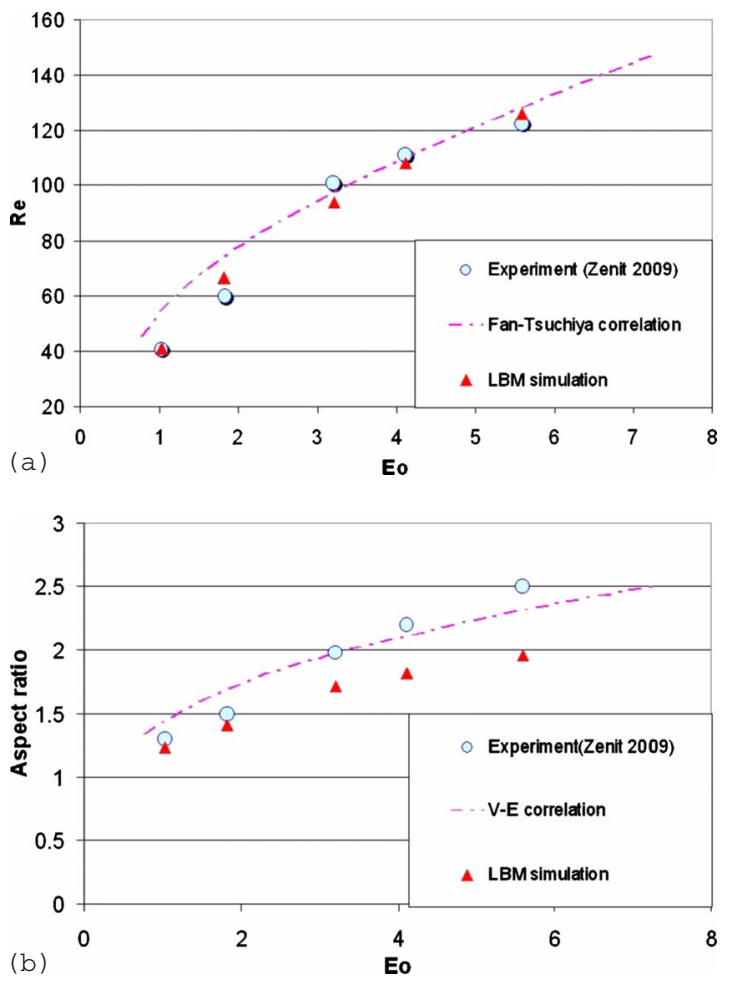

FIG. 9. (Color online) (a) Reynolds numbers for 1.5-3.5 mm air bubbles in silicone oil DMST-05. (b) Aspect ratios of 1.5-3.5 mm air bubbles in silicone oil DMST-05.

$$
u_{b}=\left(\frac{\sigma g}{\rho}\right)^{1 / 4}\left[\left(\frac{\mathrm{Eo}}{K_{b} \mathrm{Mo}^{1 / 4}}\right)^{-n}+\left(\frac{2 C}{\mathrm{Eo}^{1 / 2}}+\frac{\mathrm{Eo}^{1 / 2}}{2}\right)^{-n / 2}\right]^{-1 / n} .
$$

In the above equation, $K_{b}, C$, and $n$ are parameters determined by the liquid property and surface condition, and are chosen to be $15,1.2$, and 1.0 , respectively

The bubble shape under the simulated conditions is in the ellipsoidal regime. The bubble shape can be characterized by the aspect ratio, which is the bubble width divided by bubble height. Various experimental correlations exist for the prediction of aspect ratio in different liquids. For example, the Vakhrushev-Efremov [34] correlation gives the relation between aspect ratio and the Tadaki number (Ta) in the following form:

$$
b / h=\left\{0.81+0.2 \tanh \left[1.8\left(0.4-\log _{10} \mathrm{Ta}\right]\right\}^{-3}\right.
$$

where Ta given by

$$
\mathrm{Ta}=g^{1 / 4}\left(\frac{\rho_{l}}{\sigma}\right)^{3 / 4} d_{e} u_{b}
$$

In order to compare it with the simulation results, the terminal velocities measured from experiment [32] are used in Eq. (34) and (35) to calculate the aspect ratio. The correlation prediction for the aspect ratio is plotted as a function of the Eo number rather than the Ta number for better comparison, as seen in Fig. 9(b). In general, the simulation, experiment, and correlation give similar bubble shapes, although the simulated aspect ratio is slightly smaller than the experimen-
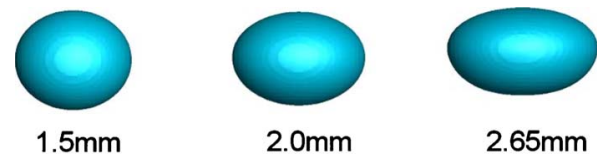

$2.65 \mathrm{~mm}$


FIG. 10. (Color online) Bubble shape corresponding to the simulation results in Fig. 9.

tal results and the correlation. Particularly, the agreement between the simulation and experiment is good for small Eo numbers, but the difference grows as Eo increases. For the largest bubble with Eo=5.6, the simulation under predicts the bubble shape by $20 \%$. The simulated bubble shapes for the five bubble sizes are shown in Fig. 10. It should be noted that the bubble dynamics in a low-viscosity liquid is in fact an extremely complicated phenomenon. For example, both the bubble surface condition and the initial bubble shape are found to have significant effect on the bubble behavior. While a bubble in a clean liquid and with a large initial shape disturbance will have a higher velocity and larger aspect ratio, the bubble of same size in the same liquid with contaminated bubble surface or small initial distortion usually has a lower velocity and more spherical shape [35]. In the current simulations, the bubbles rise with initially spherical shapes, which might be the reason for their smaller aspect ratio compared to the experiment.

Figure 11 shows the simulated shape and terminal velocity of $0.5-5 \mathrm{~mm}$ air bubbles in water. The corresponding Eo numbers are in the range between 0.034 and 3.37, while the resulted Re numbers vary from 35 to 1030. In literature, there is considerable scattering of the experimentally data for the terminal velocity for millimeter bubbles in water, mostly due to the different degree of contamination on the gas-liquid interface. As a comparison, the experimentally observed

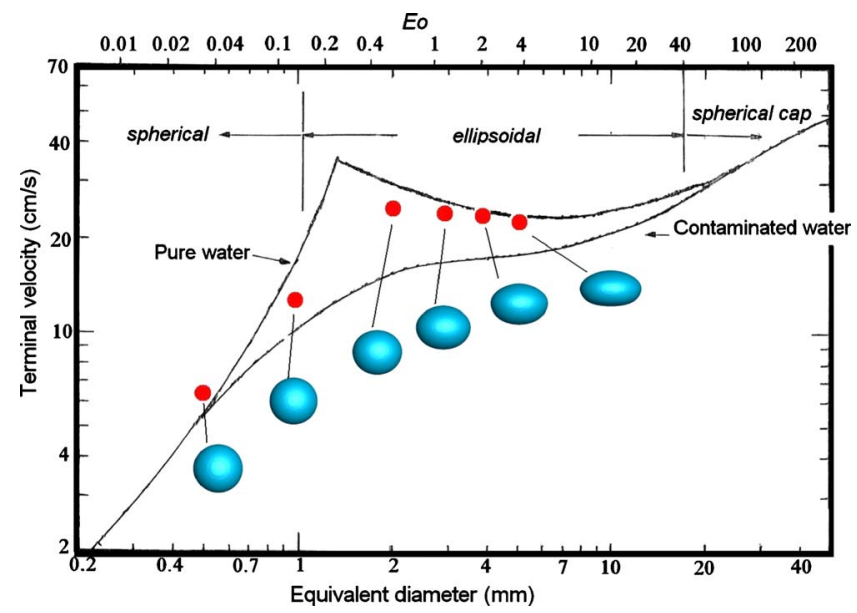

FIG. 11. (Color online) Terminal velocity and shape of 0.5-5 $\mathrm{mm}$ air bubble in water. Simulations are performed using the twocomponent MRT-LBM model. The solid curves for bubble velocity in pure and contaminated water are from [33] 

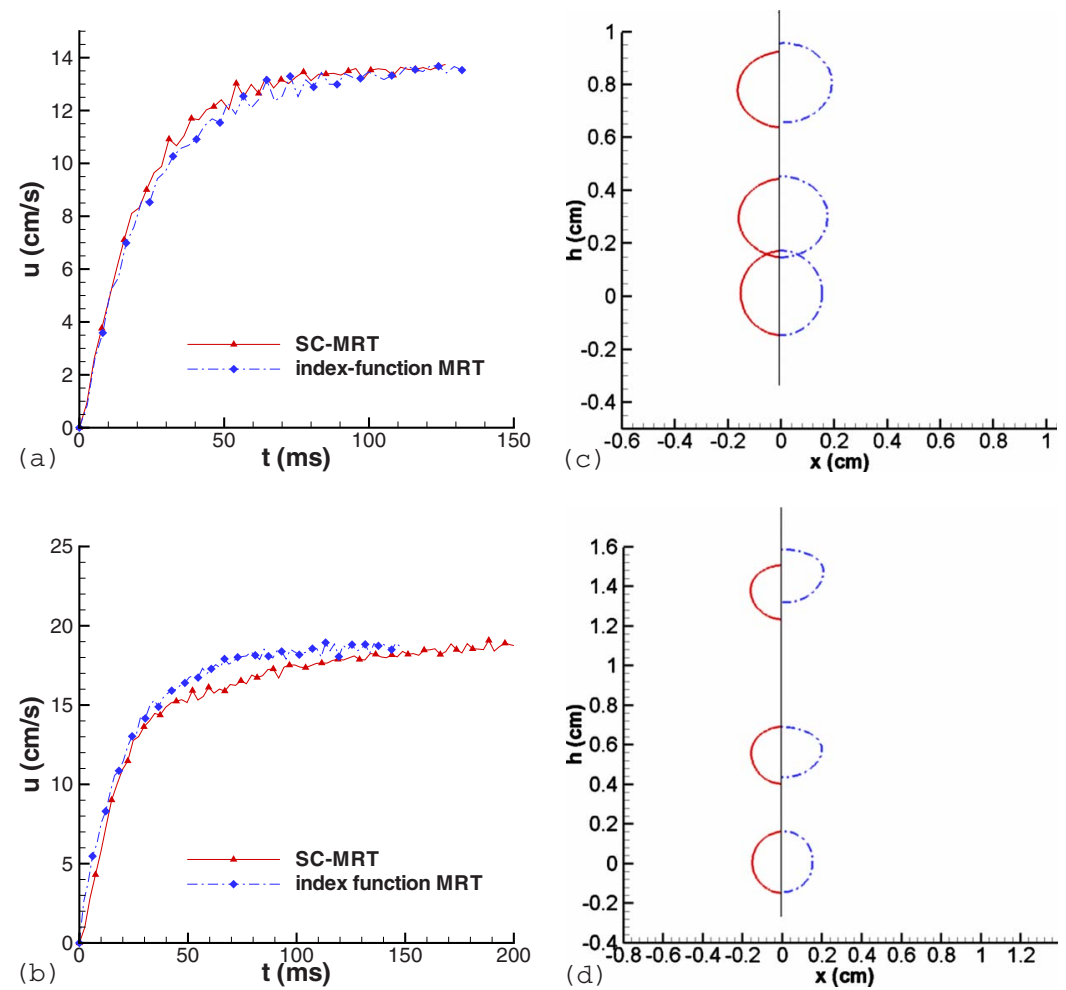

(d)

$x(\mathrm{~cm})$
FIG. 12. (Color online) Buoyant rise of a bubble in 2D. (a) Rise velocity for $\mathrm{Mo}=2.3$ $\times 10^{-5}, \mathrm{Eo}=1.3$. (b) Rise velocity for $\mathrm{Mo}=2.56$ $\times 10^{-11}, \mathrm{Eo}=1.2$. (c) and (d) Bubble shapes predicted by SC-MRT (left) and index-function MRT (right) corresponding to case (a) and (b), respectively. bubble velocities in pure and contaminated water are plotted in Fig. 11 [36]. The MRT-LBM simulation results are found to fall between the upper and lower limits of the experimentally measured velocities. For bubbles larger than $3 \mathrm{~mm}$, the simulation results are very close to the pure water condition. The bubble near $2 \mathrm{~mm}$ are usually more difficult to simulate accurately, because they are found to experience a transition in their flow pattern, which changes from a rectilinear path to a zigzag or spiral path. The transition is a complex phenomenon that is affected by many factors, and to capture the condition for the onset of the oscillation requires further investigation. In fact, no oscillation behavior is observed for the bubbles in the current 3D simulations, although a high $\mathrm{Re}$ number of $10^{3}$ is reached. The aspect ratio of the bubble is also found to be smaller than the experimental results [37], as in the previous case of bubbles in silicone oil. The smaller aspect ratio may help to explain the absence of oscillation in the current simulation, as indicated by a recent experimental study [32].

Further, to compare the performance of the current MRT approach with that of the index-function based MRT approach, the same bubble rise problems are simulated with both techniques in $2 \mathrm{D}$, and results for the rise velocity and evolution of bubble shape are presented in Fig. 12. In the first case $\left(\mathrm{Mo}=2.3 \times 10^{-5}, \mathrm{Eo}=1.3\right)$, the numerical value of surface tension in lattice units are 0.0446 and 0.0015 for the interaction potential MRT model and index-function MRT model, respectively. In order to have the same Mo and Eo numbers, the viscosity is found to be 0.0694 and 0.0225 in the two approaches according to the scaling, and a smaller buoyancy force also needs to be applied for the indexfunction method. The scaling analysis shows that the physical time step $\Delta t$ in the index-function approach is about half of that in the interaction potential approach, and therefore the index-function model requires a significantly longer simulation time to achieve the same physical time. The steady state Re predicted by the two approaches are 14.3 and 14.1, and the predicted history of the bubble velocity is also found to be similar. Both models predict a slightly deformed ellipsoidal shape of the bubble, as shown in Fig. 12(b). The second case $\left(\mathrm{Mo}=2.56 \times 10^{-11}, \mathrm{Eo}=1.2\right)$ corresponds to a $3 \mathrm{~mm}$ air bubble in water. The same surface tension values are used as in the previous case, and the viscosity is calculated to be 0.0023 and 0.00081 , leading to a time step of 0.013 and $0.0067 \mathrm{~ms}$ for the interaction potential MRT and indexfunction MRT, respectively. In both methods, the bubble reaches an almost steady velocity after about $150 \mathrm{~ms}$, which is close to that obtained using front tracking method [38]. The velocity and bubble shape simulated by the two MRTLBM methods are again similar, except that the indexfunction approach predicts a slightly higher velocity and larger deformation between 50 and $150 \mathrm{~ms}$. It is noted that the velocity and aspect ratio predicted in the above $2 \mathrm{D}$ simulations using both techniques are smaller compared to the 3D results in Fig. 11 as well as those simulated by the 3D front tracking method [38]. Compared to the index-function MRT approach, the current interaction potential MRT approach has the advantage of larger time steps and requires less computation time for high-surface tension (high Mo) flows. On the other hand, the index-function approach is more suitable for lower surface tension (high We) flows such as those involving severe deformation of the interface during droplet collision (We 8000) [39], which would be difficult for the current interaction potential approach.

\section{CONCLUSIONS}

In this study, a two-phase MRT-LBM based on the interaction potential model is developed. The new MRT-LBM 
approach employs the same interaction force calculated from the pseudopotential as that in the classic Shan-Chen model. However, the interaction force is directly incorporated into the LBM algorithm without shifting the equilibrium velocity. The collision step is performed in the moment space, and relaxation time scales for different physical quantities are decoupled to result in enhanced numerical stability. It should be pointed out that although the present method shares a similar general form with some existing two-phase MRT algorithms, they have different physical models for the phase interaction. For example, the index-function MRT scheme takes a macroscopic approach to specify the phase distribution using a scalar function which is convected by the flow, and this idea is similar to the traditional interface-capturing techniques such as the level-set method. Meanwhile in the present interaction potential MRT method the phase separation is induced spontaneously by the microscopic particle interaction which is related to the equation of state, without the need for another equation to describe the transport of the interface. Numerical simulations for two-phase flows are first conducted under the equilibrium conditions, and the results from the new MRT-LBM model are compared to those obtained from BGK-LBM models. It is found that the physical properties, such as equilibrium density, equilibrium pressure, and surface tension are not affected by the application of multiple relaxation times. On the other hand, the adjustable relaxation parameters indeed influence the numerical stability of the algorithm. By optimizing these adjustable relaxation parameters, the numerical stability of the method can be improved appreciably, and the spurious velocity can be reduced significantly. The multirange interactions for force evaluation can also be readily adopted in the current MRT-
LBM model to further reduce the magnitude of spurious velocity. To validate the MRT-LBM for dynamic flow conditions, 3D simulations are performed to investigate the buoyant rise of gas bubbles in low-viscosity liquids. Millimeter bubbles in silicone oil DMST-05 $\left(\mathrm{Mo} \sim 10^{-7}\right)$ and water $\left(\mathrm{Mo} \sim 10^{-11}\right)$ are studied with Reynolds number up to $10^{3}$. The simulated bubble shape and velocity are in good agreement with experimental observations and empirical correlations in the literature. This new MRT-LBM shows great potential in simulating many high Reynolds number twophase flows that are difficult for the traditional multiphase BGK-LBM techniques. Compared to the index-function MRT-LBM model, the present interaction potential MRTLBM model can reach a higher surface tension, and is more efficient in simulating high Mo number flows. On the other hand, the index-function MRT-LBM can maintain a sharp interface at low surface tension, and is more efficient in simulating high Eo or We flows. Although the limitations in high Eo/We or Mo numbers in the respective model can be partially alleviated by using a refiner grid in the interaction potential model or a smaller time step in the index-function model, such treatment will inevitably increase the computation cost. It should also be noted that the density ratio in each model can be significantly improved to as high as $\mathrm{O}\left(10^{3}\right)$, for example, by using different equation of state in the interaction potential model or the pressure evolution for the indexfunction model $[31,15]$.

\section{ACKNOWLEDGMENT}

This work was supported in part by an allocation of computing time from the Ohio Supercomputer Center.
[1] X. Shan and H. Chen, Phys. Rev. E 47, 1815 (1993).

[2] M. R. Swift, E. Orlandini, W. R. Osborn, and J. M. Yeomans, Phys. Rev. E 54, 5041 (1996).

[3] X. He, S. Chen, and R. Zhang, J. Comput. Phys. 152, 642 (1999).

[4] V. Sofonea, A. Lamura, G. Gonnella, and A. Cristea, Phys. Rev. E 70, 046702 (2004).

[5] G. Gonnella, A. Lamura, and V. Sofonea, Phys. Rev. E 76, 036703 (2007).

[6] K. Sankaranarayanan, X. Shan, I. G. Kevrekidis, and S. Sundaresan, J. Fluid Mech. 452, 61 (2002).

[7] T. Inamuro, T. Ogata, S. Tajima, and N. Konishi, J. Comput. Phys. 198, 628 (2004).

[8] A. Dupuis, and B. Chopard, Phys. Rev. E 67, 066707 (2003).

[9] S. Ansumali and I. V. Karlin, Phys. Rev. E 65, 056312 (2002).

[10] P. Lallemand and L.-S. Luo, Phys. Rev. E 68, 036706 (2003).

[11] J. Tolke, S. Freudiger, and M. Krafczyk, Comput. Fluids 35, 820 (2006).

[12] A. K. Gunstensen and D. Rothman, Phys. Rev. A 43, 4320 (1991).

[13] M. E. McCracken and J. Abraham, Phys. Rev. E 71, 036701 (2005).

[14] K. N. Premnath and J. Abraham, J. Comput. Phys. 224, 539 (2007).
[15] S. Mukherjee and J. Abraham, Comput. Fluids 36, 1149 (2007).

[16] T. Lee and C.-L. Lin, J. Comput. Phys. 206, 16 (2005).

[17] A. Fakhari and M. H. Rahimian, Phys. Rev. E 81, 036707 (2010).

[18] A. Kuzmin, A. A. Mohamad, and S. Succi, Int. J. Mod. Phys. C 19, 875 (2008).

[19] X. Frank, D. Funfschilling, N. Midoux, and H. Z. Li, J. Fluid Mech. 546, 113 (2006)

[20] I. O. Kurtoglu and C. L. Lin, Numer. Heat Transfer, Part B 50, 333 (2006).

[21] C. Theodoropoulos, K. Sankaranarayanan, S. Sundaresan, and I. G. Kevrekidis, Chem. Eng. Sci. 59, 2357 (2004).

[22] A. Gupta and R. Kumar, Int. J. Heat Mass Transfer 51, 5192 (2008).

[23] X. Shan, Phys. Rev. E 77, 066702 (2008).

[24] M. Sbragaglia, R. Benzi, L. Biferale, S. Succi, K. Sugiyama, and F. Toschi, Phys. Rev. E 75, 026702 (2007).

[25] X. Shan, Phys. Rev. E 73, 047701 (2006).

[26] H. Li, C. Pan, and C. T. Miller, Phys. Rev. E 72, 026705 (2005).

[27] X. He, X. Shan, and G. D. Doolen, Phys. Rev. E 57, R13 (1998).

[28] Z. Guo, C. Zheng, and B. Shi, Phys. Rev. E 65, 046308 
(2002).

[29] Z. Yu and L.-S. Fan, J. Comput. Phys. 228, 6456 (2009).

[30] E. Aulisa, S. Manservisia, and R. Scardovelli, Comput. Methods Appl. Mech. Eng. 195, 6239 (2006).

[31] P. Yuan and L. Schaefer, Phys. Fluids 18, 042101 (2006).

[32] R. Zenit and J. Magnaudet, Phys. Fluids 20, 061702 (2008).

[33] L.-S. Fan and K. Tsuchiya, Bubble Wake Dynamics in Liquids and Liquid-Solid Suspensions (Butterworth-Heinemann, Boston, 1990).

[34] I. A. Vakhrushev, and G. I. Efremov, Chem. Techn. Fuel Oils (USSR) 6, 376 (1970).
[35] M. Wu and M. Gharib, Phys. Fluids 14, L49 (2002).

[36] R. Clift, J. R. Grace, and M. E. Weber, Bubbles, Drops and Particles (Academic, New York, 1978).

[37] C. Veldhuis, A. Biesheuvel, and L. van Wijngaarden, Phys. Fluids 20, 040705 (2008).

[38] W. Dijkhuizen, E. I. V. van den Hengel, N. G. Deen, M. van Sint Annaland, and J. A. M. Kuipers, Chem. Eng. Sci. 60, 6169 (2005).

[39] S. Mukherjee and J. Abraham, Phys. Fluids 19, 052103 (2007). 